\title{
Uso de 2,4-D como regulador de crescimento e de fertilizante foliar com aminoácido
}

\section{na cultura do feijão}

\author{
Use of 2,4-D as a growth regulator and foliar fertilizer with amino acid in bean culture \\ Uso de 2,4-D como regulador del crecimiento y fertilizante foliar con aminoácidos en cultivo de \\ frijol
}

Recebido: 25/03/2021 | Revisado: 04/04/2021 | Aceito: 08/04/2021 | Publicado: 19/04/2021

Natália Bernardes Vieira

ORCID: https://orcid.org/0000-0003-2680-0543 Universidade Federal de Mato Grosso do Sul, Brasil

E-mail: nataliabernardesvieira@ hotmail.com

Sebastião Ferreira de Lima

ORCID: https://orcid.org/0000-0001-5693-912X

Universidade Federal de Mato Grosso do Sul, Brasil

E-mail: sebastiao.lima@ufms.br

Lucymara Merquides Contardi

ORCID: https://orcid.org/0000-0002-5418-5077

Universidade Estadual Paulista, Brasil

E-mail: lu_contardi@hotmail.com

Maria Gabriela de Oliveira Andrade

ORCID: https://orcid.org/0000-0002-3348-5150

Universidade Estadual Paulista, Brasil

E-mail: gabriela13andrade@ hotmail.com

Breno de Oliveira Reis

ORCID: https://orcid.org/0000-0002-6481-739X

Universidade Federal de Mato Grosso do Sul, Brasil

E-mail: brenooliveira2014ms@gmail.com

Vitória Fátima Bernardo

ORCID: https://orcid.org/0000-0002-1955-333X

Universidade Federal de Mato Grosso do Sul, Brasil

E-mail: vfbernardo@hotmail.com

Mariele Silva Abreu

ORCID: https://orcid.org/0000-0003-4719-3375 Universidade Federal de Mato Grosso do Sul, Brasil

E-mail: marielesabreu@gmail.com

Silvia Elena Navarrete Thomé

ORCID: https://orcid.org/0000-0001-8880-5337

Universidade Federal de Mato Grosso do Sul, Brasil

E-mail: silviaenthome@gmail.com

Cristieli Oliveira Vanzo

ORCID: https://orcid.org/0000-0002-3143-2485

Universidade Federal de Mato Grosso do Sul, Brasil

E-mail: cristieli.vanzo99@hotmail.com

\begin{abstract}
Resumo
A cultura do feijoeiro tem potencial para ganhos de produtividade de grãos, no entanto, não tem sido alcançado no campo. O uso de insumos como regulador de crescimento e fertilizantes foliares associado a aminoácidos pode contribuir para atingir essa meta. Assim, nesse trabalho, objetivou-se avaliar a influência da aplicação de fertilizante foliar contendo aminoácidos e do herbicida 2,4-D em subdoses, como regulador de crescimentos, na cultura do feijão carioca. $\mathrm{O}$ delineamento experimental utilizado foi em blocos casualizados com 8 tratamentos e 5 repetições. Os tratamentos foram constituídos pelas combinações da aplicação do fertilizante foliar + aminoácido e o herbicida 2,4-D em subdoses, nos estádios de desenvolvimento $\mathrm{V}_{4}, \mathrm{~V}_{6} \mathrm{e}$ em pré-floração, respectivamente. Foram avaliadas a altura de plantas, altura da inserção da primeira vagem, número de vagens por planta, número de grãos por vagem, massa de grãos por planta, número de plantas por parcela, produtividade e massa de cem grãos. O uso de fertilizante foliar + aminoácido promoveu maior altura de plantas e inserção de primeira vagem, resultou em ganhos de $8,7 \%$ e 9,1\% para número de vagens por planta e massa de grão por planta, respectivamente. A produtividade de grãos também superou a testemunha, conseguindo ganhos de 19,5\%, que representa 333, $4 \mathrm{~kg}$ a mais de grãos. A aplicação de 2,4-D resultou em redução no porte da planta, mas não incrementou a produtividade de grãos.
\end{abstract}


Palavras-chave: Produtividade de grãos; Fitohormônios; Nutrição do feijoeiro.

\begin{abstract}
The bean culture has potential for grain productivity gains, however, it has not been achieved in the field. The use of inputs as a growth regulator and foliar fertilizers associated with amino acids can contribute to achieving this goal. The aim was to evaluate the influence of the application of foliar fertilizer containing amino acids and the herbicide 2,4-D in underdoses, as a growth regulator, in the culture of carioca beans. The experimental design used was in randomized blocks with 8 treatments and 5 repetitions. The treatments were constituted by the combinations of the application of leaf fertilizer + amino acid and the herbicide 2,4-D in underdoses, in the stages of development V4, V6 and in pre-flowering, respectively. Plant height, height of insertion of the first pod, number of pods per plant, number of grains per pod, mass of grains per plant, number of plants per plot, productivity and mass of one hundred grains were evaluated. The use of foliar fertilizer + amino acid promoted greater plant height and insertion of the first pod, resulting in gains of $8.7 \%$ and $9.1 \%$ for the number of pods per plant and grain mass per plant, respectively. Grain productivity also surpassed the control, achieving gains of $19.5 \%$, which represents $333,4 \mathrm{~kg}$ more grains. The application of 2,4-D resulted in a reduction in plant size, but did not increased grain yield.
\end{abstract}

Keywords: Grain productivity; Phytohormones; Bean nutrition.

\title{
Resumen
}

El cultivo de frijol tiene potencial para ganancias de productividad de grano, sin embargo, no se ha logrado en el campo. El uso de insumos como regulador del crecimiento y fertilizantes foliares asociados con aminoácidos puede contribuir a lograr este objetivo. Así, en este trabajo, el objetivo fue evaluar la influencia de la aplicación de fertilizantes foliares que contienen aminoácidos y el herbicida 2,4-D en subdosis, como regulador del crecimiento, en el cultivo de frijol carioca. El diseño experimental utilizado fue en bloques aleatorizados con 8 tratamientos y 5 repeticiones. Los tratamientos estuvieron constituidos por las combinaciones de la aplicación de fertilizante foliar + aminoácido y el herbicida 2,4-D en subdosis, en las etapas de desarrollo V4, V6 y pre-floración, respectivamente. Se evaluaron la altura de la planta, la altura de inserción de la primera vaina, la cantidad de vainas por planta, la cantidad de granos por vaina, la masa de granos por planta, la cantidad de plantas por parcela, la productividad y la masa de cien granos. El uso de fertilizante foliar + aminoácido promovió una mayor altura de la planta y la inserción de la primera vaina, lo que resultó en ganancias de $8.7 \%$ y $9.1 \%$ para el número de vainas por planta y masa de grano por planta, respectivamente. La productividad de granos también superó el control, logrando ganancias de $19.5 \%$, lo que representa 333, 4 kg más de granos. La aplicación de 2,4-D resultó en una reducción en el tamaño de la planta, pero no aumentado el rendimiento de grano.

Palabras clave: Productividad de granos; Fitohormonas; Nutrición de frijol.

\section{Introdução}

O Brasil é o terceiro maior produtor mundial de feijão, fazendo parte dos $57 \%$ ou 15,3 milhões de toneladas de produção (Faostat, 2019), e a cultura desempenha o papel importante no sistema socioeconômico, por ser fontes de proteína e energia para grande parte da população (Souza \& Wander, 2014).

Para uma alta produtividade e rentabilidade as culturas necessitam da capacidade produtiva dos solos, associadas às práticas agrícolas, tais como: a calagem, a rotação de culturas e adubação, visando o crescimento radicular adequado da cultura (Silva \& Wander, 2013).

Dentre os produtos disponíveis no mercado, o uso de reguladores de crescimento se destaca, pois atuam como sinalizador químico e no desenvolvimento de plantas. Ligam-se em receptores nas plantas e desencadeiam várias mudanças celulares, afetando a modificação ou iniciação do desenvolvimento de tecidos ou órgãos. Os reguladores que reduzem o porte das plantas geralmente são antagonistas às giberelinas e alteram o metabolismo das mesmas (Rodrigues et al., 2003).

Existem vários produtos disponíveis no mercado para uso como regulador de crescimento, entre os quais, podem ser utilizados alguns herbicidas em subdoses, que passam a atuar como reguladores de crescimento, cessando o crescimento em altura da planta e induzindo as ramificações laterais, o que pode ocasionar a maior produção de nós reprodutivos com capacidade maior de produção de flores e de vagens por planta (Foloni et al., 2016).

Plantas que possuem dominância apical sofrem o efeito de decapitação, pois inibe o crescimento da gema terminal, assim a concentração maior da planta será no desenvolvimento das gemas laterais diminuindo o crescimento em altura e 
tornando sua arquitetura mais arbustiva (Taiz et al., 2017). A influência do 2,4-D será dependente do genótipo utilizado, do porte da planta, da concentração aplicada, do estádio de desenvolvimento da cultura e das condições de solo e clima. Assim, o regulador de crescimento passa a ser interessante quando se deseja ampliar o potencial produtivo da cultura sem que haja a necessidade da adição de novas cultivares ou adubação, com significativa redução no custo de produção (Dourado Neto et al., 2014).

O herbicida 2,4-D além de fazer o controle das plantas daninhas dicotiledôneas, também é uma auxina sintética que funciona como um regulador de crescimento, em doses baixas (Mortensen et al., 2012). Nas plantas, o 2,4-D gera uma concentração de etileno e ácido abscísico (ABA), que são provenientes da produção de espécies reativas ao oxigênio, responsáveis pelo estresse oxidativo (Grossmann, 2010).

Dentro das práticas agrícolas, outros insumos podem melhorar o desempenho da cultura do feijoeiro, entre eles destacam-se o uso de fertilizantes foliares e aminoácidos, que podem ser aplicados isoladamente ou em conjunto. A aplicação de fertilizantes foliares pode suprir o déficit nutricional dos macros ou micronutrientes no estádio vegetativo e reprodutivo da planta, com a finalidade de elevar o seu potencial produtivo (Malavolta, 2006). Os nutrientes aplicados via foliar podem propiciar efeitos diretamente no crescimento, e consequentemente na qualidade das sementes do feijoeiro (Oliveira et al., 2015).

Os aminoácidos, por sua vez, podem atuar na síntese de proteínas, no preparo de substâncias reguladoras do metabolismo vegetal e como ativador fisiológico. Além disso, interagem com a nutrição das plantas, aumentando a sua eficiência de absorção, transporte e assimilação de nutrientes (Castro \& Carvalho, 2014). As plantas são capazes de produzir esses aminoácidos quando estão sujeitas a condições de estresses, causados pelo ataque de pragas, seca, chuva entre outros fatores. Assim, a aplicação de aminoácidos sintéticos e de fertilizantes, concomitantemente, pode proporcionar melhores condições para o desenvolvimento da planta, principalmente em condições estresses (Mógor, 2015).

Considerando o potencial que os reguladores de crescimento, fertilizantes foliares e aminoácido têm sobre o crescimento e desenvolvimento das plantas de feijão, esse trabalho objetivou avaliar a influência da aplicação de fertilizante foliar contendo aminoácidos e do herbicida 2,4-D em subdoses, como regulador de crescimentos, na cultura do feijão carioca.

\section{Metodologia}

$\mathrm{O}$ experimento foi realizado em campo, em cultivo de sequeiro. A estrutura experimental foi de natureza qualitativa e quantitativa e aplicou métodos estatísticos para a avaliação dos dados coletados (Pereira et al., 2018). O experimento foi conduzido no município de Chapadão do Céu, GO, 18³7'52.6'S e 52 48'05.4' 'W com altitude média de 840 metros. De acordo com a classificação de Köppen, predomina o clima de tipo tropical úmido Aw, com a estação seca no inverno e chuvosa no verão. A determinação da precipitação na área experimental foi obtida por meio de pluviômetro, avaliado durante todo período de condução (Figura 1). 
Figura 1. Dados de precipitação $(\mathrm{mm})$, a partir de 05 de março até 15 de maio, e temperatura $\left({ }^{\circ} \mathrm{C}\right)$ na safra 2020 , para a cultura do feijão. Chapadão do Céu, GO.

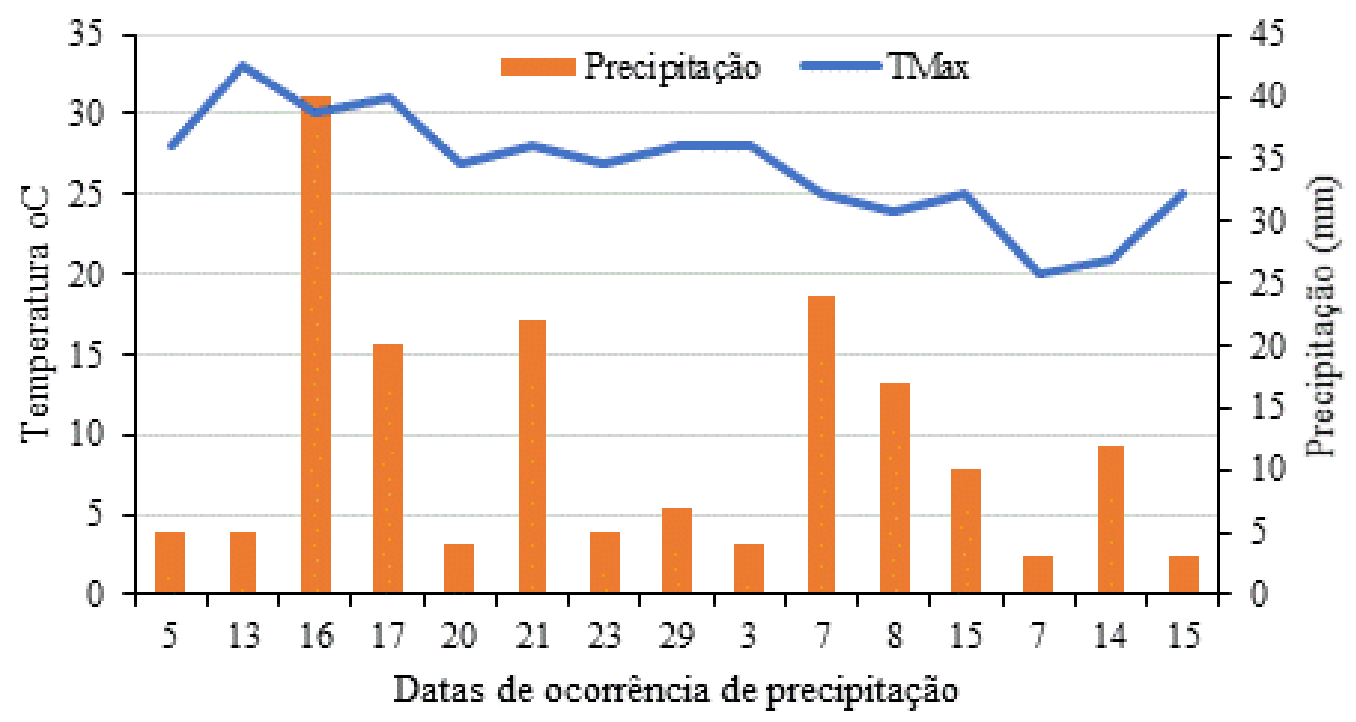

Fonte: Inmet e autores.

Durante o período de cultivo, a precipitação mínima foi de $3 \mathrm{~mm}$ e a máxima de $40 \mathrm{~mm}$, acumulando um total de 181 $\mathrm{mm}$, ficando abaixo do que é necessário para completar o ciclo com potencial de alta produtividade. Estima-se que o consumo hídrico seja de 300 a $600 \mathrm{~mm}$ ao longo de seus estádios de desenvolvimento, consumindo em média, 3 a 4 mm por dia (Marco et al., 2012).

O solo da área experimental foi classificado como Latossolo Vermelho distrófico, de textura argilosa (Santos et al., 2018). Foram determinadas em laboratório as seguintes características químicas: $\mathrm{pH}=5,1 ; \mathrm{M} . \mathrm{O} .=37,40 \mathrm{~g} \mathrm{dm}{ }^{-3}$; $\mathrm{P}, \mathrm{K}, \mathrm{Ca}$ e $\mathrm{S}=$ 14,$0 ; 0,20 ; 3,5 ; 1,30$ e 10,4 $\mathrm{mg} \mathrm{dm}^{-3} \mathrm{cmol}_{\mathrm{c}} \mathrm{dm}^{-3}$, respectivamente, $\mathrm{B}=0,30 \mathrm{mg} \mathrm{dm}^{-3}, \mathrm{Cu}=0,90 \mathrm{mg} \mathrm{dm}^{-3}, \mathrm{Mn}=22,70 \mathrm{mg} \mathrm{dm}^{-3}$, $\mathrm{Zn}=9,0 \mathrm{mg} \mathrm{dm}^{-3}, \mathrm{CTC}=11,10 \mathrm{cmol}_{\mathrm{c}} \mathrm{dm}^{-3}$.

O delineamento experimental utilizado foi em blocos casualizados com oito tratamentos e cinco repetições, totalizando 40 parcelas com 5 linhas de $5 \mathrm{~m}$ de comprimento, espaçadas de 0,45 m entre si. Para a área útil foram consideradas três linhas centrais descartando-se $0,5 \mathrm{~m}$ de cada extremidade. Os tratamentos foram constituídos pela aplicação de aminoácidos + fertilizantes no estádio fenológico V4 (28 dias após a emergência - DAE) e ou V6 (33 DAE) e aplicação de 2,4D em pré- floração (47 DAE) (Tabela 1). 
Research, Society and Development, v. 10, n. 4, e49110414262, 2021

(CC BY 4.0) | ISSN 2525-3409 | DOI: http://dx.doi.org/10.33448/rsd-v10i4.14262

Tabela 1. Tratamentos utilizados no experimento.

\begin{tabular}{cc}
\hline Tratamentos & Descrição dos tratamentos \\
\hline Testemunha & Sem aplicação \\
\hline $00-00-2,4-D$ & Aplicação de 2,4-D em pré-floração \\
\hline $00-$ V6-00 & Aplicação de Aminoseed Plus no estádio fenológico V6 \\
\hline 00-V6-2,4-D & Aplicação de Aminoseed Plus no estádio fenológico V6 e Aplicação de 2,4-D em \\
pré-floração \\
\hline V4-00-00 & Aplicaçãão de Aminoseed Plus no estádio fenológico V4 e Aplicação de 2,4-D em \\
\hline v4-00-2,4-D & pré-floração \\
\hline V4-V6-00 & Aplicação de Aminoseed Plus no estádio fenológico V4 e Aplicação de Aminoseed \\
& Plus no estádio fenológico V6 \\
\hline V4-V6-2,4-D & Aplicação de Aminoseed Plus no estádio fenológico V4, Aplicação de Aminoseed \\
& Plus no estádio fenológico V6 e Aplicação de 2,4-D em pré-floração
\end{tabular}

Fonte: Autores.

A semeadura foi realizada no dia 07 de março de 2020, utilizando a cultivar carioca TAA DAMA, que apresenta ciclo intermediário do tipo III (prostrado). A inoculação e os tratamentos químicos via semente consistiram na aplicação dos produtos Bioma soja (128 mL $\left.\mathrm{mL}^{-1} \mathrm{ha}^{-1}\right)$, Bioma milho (128 $\left.\mathrm{mL}^{-1} \mathrm{ha}^{-1}\right)$, o fungicida Derosal Plus $\left(300 \mathrm{~mL}^{-1} 100 \mathrm{~kg} \mathrm{semente}^{-1}\right)$, inseticida Cruiser $\left(500 \mathrm{~mL}^{-1} 100 \mathrm{~kg} \mathrm{semente}^{-1}\right.$ ), enraizante Aminoseed plus (aplicado na dose de $500 \mathrm{~mL}^{-1} 100 \mathrm{~kg} \mathrm{semente}^{-1}$ ) e grafite $\left(1,2 \mathrm{~kg} 100 \mathrm{~kg}\right.$ semente $\left.^{-1}\right)$.

A aplicação do fertilizante foliar Aminoseed Plus $\left(500 \mathrm{~mL} \mathrm{ha}^{-1}\right)$ e 2,4-D $\left(10 \mathrm{~mL} \mathrm{ha}^{-1)}\right.$ foi realizada com a ajuda de uma bomba costal de $18 \mathrm{~L}$ e vazão de $200 \mathrm{~L} \mathrm{ha}^{-1}$. O Aminoseed Plus é um enraizante destinado à aplicação foliar. Contém $\mathrm{N}$ solúvel em água (84 $\left.\mathrm{g} \mathrm{L}^{-1}\right), \mathrm{P}_{2} \mathrm{O}_{5}$ solúvel em água (196 $\left.\mathrm{g} \mathrm{L}^{-1}\right)$, Co teor solúvel em água $\left(7 \mathrm{~g} \mathrm{~L}^{-1}\right)$ e Mo teor solúvel em água (70 $\left.\mathrm{g} \mathrm{L} \mathrm{L}^{-1}\right)$, Metionina $(0,237 \%)$, Triptofano $(0,192 \%)$ e Ácido Glutâmico (2,967\%), Alanina (1,08\%), Arginina (0,184\%), Prolina (0,194\%), Fenilalanina (0,147\%), Lisina (0,198\%), Ácido Aspártico (1,735\%), Glicina (1,78\%) e Valina (0,264\%).

Durante o desenvolvimento da cultura foram feitos os tratos culturais via aplicações de produtos químicos para o controle de plantas daninhas, pragas e doenças (Tabela 2). 
Tabela 2. Aplicações realizadas durante o manejo da cultura do feijão, Chapadão do Céu, GO, 2020.

\begin{tabular}{|c|c|}
\hline Sequência de aplicação & Princípio ativo/Dose \\
\hline 1 & TA $3550 \mathrm{~mL}^{-1} 100 \mathrm{~L}^{-1}+$ Paraquate $200 \mathrm{~g} \mathrm{~L}^{-1}+$ Joint \\
\hline \multirow[t]{2}{*}{2} & TA $3550 \mathrm{~mL}^{-1} 100 \mathrm{~L}^{-1}+$ Acetamiprido $200 \mathrm{~g} \mathrm{~kg}^{-1}+$ Zita-Cipermetrina $200 \mathrm{~g} \mathrm{~L}^{-1}$ \\
\hline & Bifentrina $180 \mathrm{~g} \mathrm{~L}^{-1}+$ Joint + Fomesafem $250 \mathrm{~g} \mathrm{~L}^{-1}$ \\
\hline \multirow[t]{2}{*}{3} & Profoxidim $200 \mathrm{~g} \mathrm{~L}^{-1}+$ Acetamiprido $200 \mathrm{~g} \mathrm{Kg}^{-1}+$ Clorpirifós $480 \mathrm{~g} \mathrm{~L}^{-1}+$ \\
\hline & Mancozebe $800 \mathrm{~g} \mathrm{Kg}^{-1}+$ Abamectina $18 \mathrm{~g} \mathrm{~L}^{-1}$ \\
\hline \multirow[t]{2}{*}{4} & TA $3550 \mathrm{~mL}^{-1} 100 \mathrm{~L}^{-1}+$ MAP Purificado (N 12\% P 61\%) $4 \mathrm{~kg}^{-1} \mathrm{ha}^{-1}+$ \\
\hline & Multibiocomoni K 35, $84 \mathrm{~g} \mathrm{~L}^{-1}$ Co 6,40 $\mathrm{g} \mathrm{L}^{-1}$ Mo $64 \mathrm{~g} \mathrm{~L}^{-1} \mathrm{Ni} 6,40 \mathrm{~g} \mathrm{~L}^{-1}$ \\
\hline \multirow[t]{2}{*}{5} & Profoxidim $200 \mathrm{~g} \mathrm{~L}^{-1}+$ Acetamiprido $250 \mathrm{~g} \mathrm{Kg}^{-1}$ Bifentrina $250 \mathrm{~g} \mathrm{Kg}^{-1}+$ Benzoato \\
\hline & de Emamectina $200 \mathrm{~g} \mathrm{Kg}^{-1}+$ MAP Purificado + Multicomoni + Mn 10 \\
\hline \multirow[t]{4}{*}{6} & TA $3550 \mathrm{~mL}^{-1} 100 \mathrm{~L}^{-1}+$ Benzoato de Emamectina $200 \mathrm{~g} \mathrm{Kg}^{-1}+$ Clorantraniliplore \\
\hline & $200 \mathrm{~g} \mathrm{~L}^{-1}+$ Clorotalonil $500 \mathrm{~g} \mathrm{~L}^{-1}+$ Tiametoxam $141 \mathrm{~g} \mathrm{~L}^{-1}$ Lambda-Cialotrina 106 \\
\hline & $\mathrm{g} \mathrm{L}^{-1}+\mathrm{Mn} 10+$ Trifloxistrobina $150 \mathrm{~g} \mathrm{~L}^{-1}$ Protioconazol $175 \mathrm{~g} \mathrm{~L}^{-1}+\mathrm{MAP}+$ \\
\hline & Tebutiurom $500 \mathrm{~g} \mathrm{~L}^{-1}$ \\
\hline 7 & TA $3550 \mathrm{~mL}^{-1} 100 \mathrm{~L}^{-1}+$ Cab dry $\left(\mathrm{K} 2 \%\right.$ Ca $20 \%$ B 4\%) $3 \mathrm{~kg} \mathrm{ha}^{-1}$ \\
\hline \multirow[t]{2}{*}{8} & TA $35+$ Lufenurom $50 \mathrm{~g} \mathrm{~L}^{-1}+$ Abamectina $18 \mathrm{~g} \mathrm{~L}^{-1}$ Diafentiurom $500 \mathrm{~g} \mathrm{~L}^{-1}+$ \\
\hline & Bifentrina $100 \mathrm{~g} \mathrm{~L}^{-1}$ Casugamicina $20 \mathrm{~g} \mathrm{~L}^{-1}$ \\
\hline 9 & TA $3550 \mathrm{~mL}^{-1} \mathrm{~L}^{-1}+$ Espinetoram $120 \mathrm{~g} \mathrm{~L}^{-1}+$ Metomil $215 \mathrm{~g} \mathrm{~L}^{-1}$ \\
\hline 10 & Benzoato de Emamectina $200 \mathrm{~g} \mathrm{Kg}^{-1}+$ Clorpifós $480 \mathrm{~g} \mathrm{~L}^{-1}$ \\
\hline
\end{tabular}

Fonte: Autores.

A colheita ocorreu $80 \mathrm{DAE}$ e as variáveis avaliadas no experimento foram: altura de plantas (ALTP) medida da base (colo) até a extremidade da planta (final do eixo caulinar), altura de inserção da primeira vagem (A1V) medida da base (colo) até a inserção da primeira vagem, número de vagens por planta (NVP) contagem de todas as vagens de cinco plantas, número de grãos por vagem (NGV), retirando-se 10 vagens a partir de cinco plantas, massa de grãos por planta (MGP), em que todas as vagens de cinco plantas foram abertas e pesadas e a umidade ajustada a 13\%, produtividade de grãos (PROD) todas as plantas das 3 linhas da parcela foram colhidas, secas e trilhadas. Determinando a massa da parcela e a umidade dos grãos e massa de cem grãos (M100) pesagem e contagem de 100 grãos, ajustando a umidade a $13 \%$.

Os dados obtidos foram submetidos à análise da variância e as médias comparadas pelo teste de Skott-Knott a 5\% de probabilidade, utilizando o programa Sisvar (Ferreira, 2011).

\section{Resultados e Discussão}

Todas as variáveis avaliadas foram influenciadas pelos tratamentos utilizados, com exceção da massa de cem grãos (Tabela 3). 
Tabela 3. Altura de plantas (ALTP), altura de inserção da primeira vagem (A1V), número de vagens por planta (NVP), número de grãos por vagem (NGV), massa de grãos por planta (MGP), massa de cem grãos (M100) e produtividade (PROD), utilizando fertilizante foliar + aminoácidos e 2,4-D, Chapadão do Céu-GO, 2020

\begin{tabular}{lcccccccc}
\hline \multicolumn{7}{c}{ Quadrado Médio } \\
\hline FV & GL & ALTP & A1V & NVP & NVG & MGP & M100 & PROD \\
\hline Bloco & 4 & 0,624 & 0,672 & 2,43 & 0,764 & 1,731 & 0,548 & 1,45 \\
Tratamentos & 7 & $28,999^{*}$ & $24,017^{*}$ & $7,186^{*}$ & $5,705^{*}$ & $8,193^{*}$ & 2,653 & $29,152^{*}$ \\
Erro & 28 & 1,0985 & 0,3757 & 0,232 & 0,1204 & 0,3122 & 0,2956 & 29,0927 \\
CV $(\%)$ & & 2,67 & 5,55 & 3,91 & 4,64 & 5,23 & 2,94 & 3,58 \\
\hline
\end{tabular}

*Significativo em nível de 5\% de probabilidade. Fonte: Autores.

A utilização do fertilizante foliar + aminoácidos em V4 e V6, sem aplicação de 2,4-D proporcionou maior altura de planta, isso ocorre pois os micronutrientes presentes nos fertilizantes foliares atuam no crescimento das plantas participando em diversos processos metabólicos, dentre eles a síntese de proteínas, hormônios e carboidratos (Rozas, 2019). Enquanto o uso de 2,4-D isolado ou com aplicação de fertilizante foliar + aminoácidos, mais tardio (V6), foram os tratamentos que resultaram em menor porte da planta (Tabela 4).

A maior altura de inserção de primeira vagem foi observada sempre na presença de fertilizante foliar + aminoácidos, não se restringindo a presença ou ausência de 2,4-D (Tabela 4). As vagens que estão mais distantes do solo favorecem o manejo fitossanitário, reduzem o contato com o solo e diminui o apodrecimento de vagens (Salgado et al., 2012). A altura de inserção das primeiras vagens está correlacionada positivamente a altura de plantas (Heiffig \& Câmera, 2006).

Tabela 4. Altura de plantas (ALTP) e altura de inserção da primeira vagem (A1V) utilizando fertilizante foliar + aminoácidos e 2,4-D, Chapadão do Céu-GO, 2020.

\begin{tabular}{ccc}
\hline Tratamentos & ALTP & A1V \\
\hline Testemunha & $89,86 \mathrm{~d}$ & $13,448 \mathrm{~b}$ \\
$00-00-2,4-D$ & $84,06 \mathrm{e}$ & $13,008 \mathrm{~b}$ \\
$00-\mathrm{V} 6-00$ & $97,62 \mathrm{~b}$ & $16,904 \mathrm{a}$ \\
00-V6-2,4-D & $86,54 \mathrm{e}$ & $17,024 \mathrm{a}$ \\
V4-00-00 & $92,796 \mathrm{c}$ & $14,236 \mathrm{~b}$ \\
V4-00-2,4-D & $90,232 \mathrm{~d}$ & $17,252 \mathrm{a}$ \\
V4-V6-00 & $102,468 \mathrm{a}$ & $16,05 \mathrm{a}$ \\
V4-V6-2,4-D & $93,68 \mathrm{c}$ & $13,252 \mathrm{~b}$
\end{tabular}

*Médias seguidas de mesma letra, na coluna, não diferem entre si pelo teste Tukey a 5\% de probabilidade. Fonte: Autores.

O uso de fertilizante foliar + aminoácidos em V6 isoladamente ou na presença de 2,4-D ou o uso de fertilizante foliar + aminoácidos em V4 isolado ou também em V6, sem aplicação de 2,4-D foram os tratamentos que resultaram em maior número de vagens por planta. Esses tratamentos proporcionam em média, um ganho de 8,7\% em vagens, quando comparado à testemunha (Tabela 5).

Para o NGV (Tabela 5), nenhum tratamento conseguiu superar a testemunha, no entanto a aplicação do fertilizante foliar + aminoácidos isoladamente no estádio de desenvolvimento V4 e V6 ou em conjunto com o 2,4-D obtiveram média 
baixa, deve-se ressaltar que essa variável, segundo Andrade et al. (1998) está relacionada com a sua alta herdabilidade genética, sendo menos afetada pelas condições ambientais.

Tabela 5. Número de vagens por planta (NVP) e número de grãos por vagem (NGV) com e sem o uso de fertilizante foliar + aminoácidos e 2,4-D, Chapadão do Céu-GO.

\begin{tabular}{ccc}
\hline Tratamentos & NVP & NGV \\
\hline Testemunha & $12,7 \mathrm{~b}$ & $6,0 \mathrm{a}$ \\
$00-00-2,4-D$ & $12,6 \mathrm{~b}$ & $5,48 \mathrm{~b}$ \\
00-V6-00 & $13,96 \mathrm{a}$ & $5,72 \mathrm{~b}$ \\
00-V6-2,4-D & $13,4 \mathrm{a}$ & $5,44 \mathrm{~b}$ \\
V4-00-00 & $13,72 \mathrm{a}$ & $5,62 \mathrm{~b}$ \\
V4-00-2,4-D & $12,56 \mathrm{~b}$ & $5,88 \mathrm{a}$ \\
V4-V6-00 & $14,12 \mathrm{a}$ & $6,20 \mathrm{a}$ \\
V4-V6-2,4-D & $13,16 \mathrm{~b}$ & $6,12 \mathrm{a}$ \\
\hline
\end{tabular}

*Médias seguidas de mesma letra, na coluna, não diferem entre si pelo teste Tukey a 5\% de probabilidade. Fonte: Autores.

A aplicação do fertilizante foliar + aminoácidos em V4, V6 ou ambos, sem uso de 2,4-D resultou em maior massa de grãos por planta (Tabela 6), superando em $9,1 \%$ a massa obtida com a testemunha. Com o fornecimento de aminoácidos ocorre um aumento na quantidade de sólidos solúveis, encontrando maiores quantidades de proteínas totais nos tecidos e assim favorecendo o enchimento de grãos, logo a planta obtém resultados superiores com a sua aplicação (Lambais, 2011). Esse aumento no acúmulo de assimilados muitas vezes está relacionado aos parâmetros fisiológicos da planta (Zobiole et al., 2011; Merotto júnior et al., 2015; Fan et al., 2017). De acordo com Castro (2009) estes compostos são capazes de agir como ativadores de enzimas, precursores de hormônios e favorecem a disponibilização de compostos capazes de proporcionar tolerância a estresses e elevar a produção.

A utilização do fertilizante foliar + aminoácidos nos estádios de desenvolvimento $\mathrm{V}_{4}$ e $\mathrm{V}_{6}$ promoveu a maior produtividade de grãos (Tabela 6), superando em 19,5\% a testemunha. Com a necessidade de aminoácidos em seu metabolismo, a partir deles é que são formadas as proteínas e fitormônios, indispensáveis no desenvolvimento das plantas, garantindo uma maior eficiência nas atividades metabólicas e consequentemente o aumento da produtividade (Braga, 2016). A disponibilidade de nutrientes, fornecido por meio da adubação foliar também é fundamental para garantir maior produtividade. Silva e Buso (2020) obtiveram maior produtividade de grãos de feijão utilizando K aplicado via foliar na dose de $0,410 \mathrm{~kg} \mathrm{ha}^{-1}$. 
Tabela 6. Comparação de médias para as seguintes variáveis, massa de grãos por planta (MGP), massa de cem grãos (M100) e produtividade com e sem o uso de fertilizante foliar + aminoácidos e 2,4-D, Chapadão do Céu-GO.

\begin{tabular}{cccc}
\hline Tratamentos & MGP $(\mathrm{g})$ & M100 $(\mathrm{g})$ & PROD $\left(\mathrm{kg} \mathrm{ha}^{-1}\right)$ \\
\hline Testemunha & $13,096 \mathrm{~b}$ & $21,92 \mathrm{a}$ & $1705,32 \mathrm{c}$ \\
00-00-2,4-D & $12,1076 \mathrm{~b}$ & $23,1 \mathrm{a}$ & $1818,89 \mathrm{c}$ \\
00-V6-00 & $13,9204 \mathrm{a}$ & $22,398 \mathrm{a}$ & $1937,89 \mathrm{~b}$ \\
00-V6-2,4-D & $12,5144 \mathrm{~b}$ & $22,494 \mathrm{a}$ & $1919,50 \mathrm{~b}$ \\
V4-00-00 & $14,1516 \mathrm{a}$ & $22,056 \mathrm{a}$ & $1753,28 \mathrm{c}$ \\
V4-00-2,4-D & $13,0944 \mathrm{~b}$ & $23,15 \mathrm{a}$ & $1552,38 \mathrm{~d}$ \\
V4-V6-00 & $14,8332 \mathrm{a}$ & $21,954 \mathrm{a}$ & $2038,74 \mathrm{a}$ \\
V4-V6-2,4-D & $13,1196 \mathrm{~b}$ & $22,608 \mathrm{a}$ & $1773,81 \mathrm{c}$
\end{tabular}

*Médias seguidas de mesma letra, na coluna, não diferem entre si pelo teste Tukey a 5\% de probabilidade. Fonte: Autores.

Pode se observar, no contexto geral, que a aplicação de fertilizante foliar + aminoácidos nos estádios de desenvolvimento $\mathrm{V}_{4}$ e $\mathrm{V}_{6}$, propiciou maior produtividade de grãos, mesmo em condições de déficit hídrico durante os dias 15 de abril a 7 de maio de 2020, fisiologicamente o feijão reduz a sua condutância estomática e aumenta a resistência difusiva ao vapor de água, reduz a transpiração e consequentemente o suprimento de $\mathrm{CO}_{2}$ para a fotossíntese, diminuindo a fixação de carbono e a produtividade de grãos (Oliveira et al., 2005). Porém a produtividade é o menos afetado, uma vez que a planta adota como estratégia a formação de poucas sementes, porém variáveis (Stone et al., 2006). Essa condição pode ter afetado o efeito do 2,4-D como regulador de crescimento benéfico à cultura do feijão. Com isso, pode-se indicar trabalhos futuros que abordem o feijão safra ou feijão irrigado, com possibilidade de maior controle na disponibilidade de água para a cultura.

\section{Conclusão}

A utilização de fertilizante foliar junto com aminoácidos promoveu incrementos na altura de plantas, inserção de primeira vagem, número de vagens por planta, massa de grãos por planta e produtividade de grãos.

A aplicação de 2,4-D propiciou redução no porte da planta, mas não resultou em maior produtividade de grãos.

Indica-se a realização de outros trabalhos em condições de cultivo sem estresse hídrico, buscando maior efeito do regulador de crescimento em condições de maior taxa de crescimento da planta.

\section{Referências}

Andrade, M., Diniz, A. R., Carvalho, J. D. \& Lima S. F. (1998). Resposta da cultura do feijoeiro à aplicação foliar de molibdênio e às adubações nitrogenadas de plantio e cobertura. Ciência e Agrotecnologia, 22(4), 499-508.

Braga, G. N. M. (2016). Na Sala com Gismonti Assuntos Sobre Agronomia. http://agronomiacomgismonti.blogspot.com.br/2011/10uso-dos-aminoacidos-estaavancanco-na.html

Castro, P. D. C. \& Carvalho, M. E. A. (2014). Aminoácidos e suas aplicações na agricultura. ESALQ/USP.

Castro, P. R. C. (2009). Princípios da fertilização foliar. FUNEP.

Dourado Neto, D., Dario, G. J. A., Barbieri, A. P. P., \& Martin, T. N. (2014). Ação de bioestimulante no desempenho agronômico de milho e feijão. Bioscience Journal, 30(1), 371-379.

Fan, H. F., Ding, L., Xu, Y. L. \& Du, C. X. Antioxidant system and photosynthetic characteristics responses to shortterm PEG-induced drought stress in cucumber seedling leaves. Russian Journal Of Plant Physiology, 64(2), 162-173. 10.1134/s1021443717020042.

FAOSTAT. (2019). Crops. http://www.fao.org/faostat/en/\#data/QC

Ferreira, D. F. (2011). Sisvar: a computer statistical analysis system. Ciência e agrotecnologia, 35(6), 1039-1042. 
Foloni, J. S. S., Henning, F. A., Mertz-Henning, L. M., Pipolo, A. E. \& Melo, C. L. P. (2016). Lactofen e etefon como reguladores de crescimento de cultivares de soja. XXXV Reunião de Pesquisa da Soja.

Grossmann, K. (2010). Auxin herbicides: current status of mechanism and mode of action. Pest Management Science, 66(2), 113-120.

Heiffig, L. S., \& Câmara, G. D. S. (2006). Soja: colheita e perdas. ESALQ.

Lambais, G. R. (2011). Aminoácidos como coadjuvantes da adubação foliar e do uso glifosato na cultura de soja. Dissertação de Mestrado. 10.11606/D.112011.tde-28062011-145152.

Malavolta, E. (2006). Manual de nutrição mineral de plantas. Agronômica Ceres.

Marco, K., Dallacort, R., Júnior, C. A. F., Sérgio, P., de Freitas, L., \& Villela, T. G. (2012). Aptidão Agroclimática e Características Agronômicas de FeijãoComum Semeado na Safra das Âguas em Tangará da Serra-MT. Enciclopédia Biosfera, 8(15), 160.

Merotto Junior, A., Wagner, J. \& Meneguzzi, C. (2015). Effects of glyphosate and foliar application of micronutrients in transgenic soybean. Bioscience Journal 31(2), 499-508.

Mógor, A. (2015). Fertilizantes foliares complexados com aminoácidos ajudam a corrigir carências nutricionais em plantas. http://www.agrolink.com.br/noticias/fertilizantes-foliares-complexados-com-aminoacidos-ajudam-a-corrigir-carencias-nutricionais-em-plantas_344608.html

Mortensen, D. A, Egan, J. F., Maxwell, B. D., Ryan, M. R. \& Smith, R. G. (2012). Navegando em um crítico para o gerenciamento sustentável de ervas daninhas. BioScience, 62 (1), 75-84.

Oliveira, A. D. D., Fernandes, E. J. \& Rodrigues, T. D. J. (2005). Condutância como indicador de estresse hídrico em feijão. Engenharia Agrícola, 25(1), 8695.

Oliveira, F. D. A. D., Medeiros, J. F. D., Alves, R. D. C., Lima, L. A., Santos, S. T. D., \& Régis, L. R. D. L. (2015). Produção de feijão caupi em função da salinidade e regulador de crescimento. Revista brasileira de engenharia agrícola e ambiental, 19(11), 1049-1056.

Pereira, A. S., Shitsuka, D. M., Parreira, F. J., \& Shitsuka, R. (2018). Metodologia da pesquisa científica. UFSM. https://repositório.ufsm.br/bitstrem/handle/1/15824/Lic_Computacao_Metodologia-Pesquisa-Cientifica. pdf.

Rodrigues, O., Didonet, A. D., Teixeira, M. C. C. \& Roman, E. S. (2003). Redutores de crescimento. Embrapa Trigo. Circular técnica, 14.

Rozas, H. R. S. (2019). Zinco: um micronutriente limitante para o milho. https://maissoja.com.br/zinco-um-micronutriente-limitante-para-o-milho/>.

Salgado, F. H. M., Silva, J., de Oliveira, T. C., Tonello, L. P., Passos, N. G. \& Fidelis, R. R. (2012). Efeito do nitrogênio em feijão cultivado em terras altas no sul do estado de Tocantins. Ambiência, 8(1), 125-136.

Santos, H. G., Jacomine, P. K. T., Anjos, L. H. C., Oliveira, V. A., Lumbreras, J. F., Coelho, M. R., Almeida, J. A., Araujo Filho, J. C., Oliveira, J. B. \& Cunha, T. J. F. (2018). Sistema Brasileiro de Classificação de Solos. Embrapa.

Silva, L. F. A. \& Buso, W. H. D. (2020). Desempenho do feijoeiro cv. Pérola submetido a doses e épocas de aplicação de potássio foliar. Research, Society and Development, 9(9), e363997298. 10.33448/rsd-v9i9.7298.

Silva, O. F. \& Wander, A. E. (2013). O feijão-comum no Brasil: passado, presente e futuro. Embrapa Arroz e Feijão-Documentos.

Souza, R. S. \& Wander, A. E. (2014). Aspectos econômicos da produção de feijão no Brasil. Revista de Política Agrícola, 23(3), 43-54.

Stone, L. F., Silveira, P. M., Moreira, J. A. A. \& Braz, A. J. B. P. (2006). Evapotranspiração do feijoeiro irrigado em plantio direto sobre diferentes palhadas de culturas de cobertura. Pesquisa Agropecuária Brasileira, 41(4), 577-582.

Taiz, L. Zeiger, E., Møller, I. M., \& Murphy, A. (2017). Fisiologia e desenvolvimento vegetal. Artmed Editora.

Zobiole, L. H. S., Kremer, R. J., Oliveira Junior, R. S., \& Constantin, J. (2011). Glyphosate affects clorophyll, nodulation and nutriente accumulation of "second generation" glyphosate-resistant soybean (Glycine max L.). Pesticide Biochemistry and Physiology, 99, 53-60. 10.1016/j.pestbp.2010.10.005. 